\title{
Assessing insulin effectiveness at the end of the day: Once-daily versus twice-daily insulin glargine injection
}

\author{
Mark R. Burge $^{1 *}$, Eric R. Schroeder ${ }^{2}$, Stephen Mitchell ${ }^{3}$ \\ ${ }^{1}$ Department of Medicine, Division of Endocrinology and Metabolism, University of New Mexico Health Sciences Center, Albu- \\ querque, USA; ${ }^{*}$ Corresponding Author: mburge@salud.unm.edu \\ ${ }^{2}$ Division of Cardiovascular Medicine, South Miami Hospital, Miami, USA \\ ${ }^{3}$ Department of Medicine, Endocrinology, The Vancouver Clinic, Vancouver, USA
}

Received 11 February 2012; revised 15 March 2012; accepted 23 April 2012

\begin{abstract}
Objective: Evidence supporting the twice-daily administration of insulin glargine as an approach to address its waning effectiveness at the end of a 24 hour period is sparse. We hypothesized that insulin concentrations determined during the last four hours of a 24 hour period would be greater when identical doses of insulin glargine were administered twice-daily as compared to once-daily among type 1 diabetes patients. Research Methods: Ten subjects with insulin deficient type 1 diabetes were admitted for two 38-hour studies at least one week apart. Patients received full-dose insulin glargine once daily at 0800 and half-dose insulin glargine twice-daily at 0800 and 2000 for at least one week in random order prior to overnight studies. Overnight glucose was stabilized with intravenous insulin on the evening prior to study, and subjects fasted and did not receive short acting insulin during the study period. Insulin concentrations were assessed every $\mathbf{3 0}$ minutes with an ultra-sensitive assay between study hours 20 and 24. Results: Insulin concentrations for the final four hours of study period did not differ between once-daily and twice-daily insulin glargine administration $(p=0.38)$. Home glucose testing results and overnight plasma glucose concentrations did not differ between study conditions. Conclusions: These results demonstrate that insulin concentrations are equivalent during the last four hours of a 24-hour period when insulin glargine is administered once- or twice-daily. These findings do not support a role for twice-daily insulin glargine in the management of patients with type 1 diabetes.
\end{abstract}

Keywords: Insulin Injection; Timing; Insulin Glargine; Basal Insulin; Type 1 Diabetes

\section{INTRODUCTION}

The first long-acting insulin analog, insulin glargine, was introduced in the United States in 2001. It has since become a basal insulin of choice due to its once-daily dosing and its reduced peak activity, resulting in fewer episodes of hypoglycemia [1,2]. Nevertheless, it is a commonly held clinical notion that once-daily insulin glargine administration provides inadequate basal coverage for some patients with type 1 diabetes. Pharmacodynamic studies are consistent with the possibility that relative insulinopenia may occur with once-daily dosing. Lepore et al. showed that the "end of action" of insulin glargine was $22 \pm 4$ hrs in subjects with type 1 diabetes [2]. Similarly, a euglycemic glucose clamp study in non-diabetic subjects showed that glucose infusion rates began to decline approximately 22 hours after insulin glargine injection [3]. Clement et al. published a case report in 2002 which reported a patient with elevated bedtime glucose values on once-daily insulin glargine therapy administered in the evening, and this patient noted significant improvement in capillary blood glucose measurements after administering insulin glargine as a split dose every 12 hours [4]. In order to examine the hypothesis that twice-daily insulin glargine administration provides superior insulinization during the waning hours of a 24-hour period, this study was designed to compare insulin and glucose concentrations with administration of equivalent daily doses of insulin glargine injected once or twice daily during a 38 hour fast.

\section{RESEARCH DESIGN AND METHODS}

\subsection{Study Subjects}

Ten subjects with type 1 diabetes mellitus (9 female) 
were studied on two separate occasions in a prospective, randomized, cross-over study. Inclusion criteria included age 18 - 50 years, diagnosis of type 1 diabetes at least one year prior to study enrollment, an A1c less than 9\%, and basal-bolus therapy using insulin glargine. Exclusion criteria included complications requiring hospitalization within the last 12 months, allergy to insulin glargine, clinically significant chronic medical disease (AST greater than 2.5 timers upper limit of normal, creatinine greater than $1.8 \mathrm{mg} / \mathrm{dl}$, hemoglobin less than 11 for males and less than 10 for females, or NYHA class III or IV heart disease), systolic and diastolic blood pressure above 180 or $110 \mathrm{mmHg}$, respectively, or active alcohol or drug abuse. The study was approved by the University of New Mexico Human Research Protections Office and all participants provided written informed consent.

\subsection{Study Protocol}

After an initial screening visit to determine eligibility and to obtain informed consent, study subjects were randomized to receive insulin glargine as either one full-dose injection ( 0.2 units $/ \mathrm{kg})$ at 2000 hours or two half-dose injections (0.1 units/kg) at 0800 and 2000 hours. Subjects subsequently recorded fasting capillary blood glucose (CBG) levels prior to breakfast and in the evenings before dinner, and insulin glargine doses were titrated until fasting CBG's were less than $150 \mathrm{mg} / \mathrm{dl}$ $(8.33 \mathrm{mmol} / \mathrm{l})$. Once the dose of study insulin remained unchanged for one week with fasting CBG's less than $150 \mathrm{mg} / \mathrm{dl}$ (8.33 mmol/l), subjects were admitted for an overnight study.

Subjects were admitted to the University of New Mexico Inpatient Clinical Research Unit at 1500 on the afternoon prior to study. In order to avoid carryover of long-acting insulin from the previous day, subjects were instructed not to take any insulin glargine at home on the day of admission. All subjects received a $7 \mathrm{kcal} / \mathrm{kg}$ American Diabetic Association meal at 1800, and then fasted for the remainder of the study [5]. A continuous intravenous infusion of human regular insulin was administered between 1900 on day 1 and 0700 on day 2 to achieve a target CBG of $90-120 \mathrm{mg} / \mathrm{dl}$ (5.0 - 6.66 $\mathrm{mmol} / \mathrm{l})$. CBG's were monitored on an hourly basis and the insulin infusion was adjusted as needed to maintain CBG's in the target range. At 0800 on day 2, subjects received either full- or half-dose insulin glargine injected subcutaneously into the abdomen, corresponding to their assigned regimen. Subjects randomized to the twicedaily insulin glargine regimen received their second injection at 2000 on day 2. Blood samples were obtained for plasma glucose and PEG-treated serum insulin every 60 minutes between 0800 on day 2 and 0400 on day 3, and every 30 minutes between 0400 and 0800 (i.e., the final 4 hours of the 24 hour period since initial insulin glargine administration) on day 3 . In addition, bedside CBG's were performed every 60 minutes. If hypoglycemia occurred, defined as a CBG value less than $50 \mathrm{mg} / \mathrm{dl}$

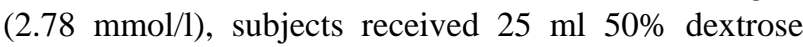
intravenously and the study was continued. The inpatient study concluded at 0800 on day 3 . At the conclusion of the first overnight stay, subjects switched to the alternative study insulin regimen (either once or twice-daily insulin glargine) and repeated the protocol as described above, followed by a second inpatient admission. Upon conclusion of the second hospital admission, subjects returned to their pre-study insulin regimen.

\subsection{Sample Analysis}

CBG concentrations were determined in duplicate using mixed venous blood with a standard, commercially available meter (Accucheck Advantage, Roach Diagnostics, Indianapolis, IN). Plasma glucose was assessed using the ACE Glucose Reagent (Alfa Wassermann, Caldwell, NJ). Free insulin concentrations were determined using an Ultra Sensitive Human Insulin Radioimmunoassay (Linco, St Charles, Missouri), which employs the double antibody/PEG technique to achieve a sensitivity of $0.2 \mathrm{mcU} / \mathrm{ml}$ when using a 100 microliter sample.

\subsection{Validation of the Insulin Glargine Assay}

Validation of the use of the Linco ultra-sensitive human insulin radioimmunoassay for insulin glargine was performed using the methods of Owen et al. [6]. The Human Insulin RIA Kit from Linco Research (St. Charles, Missouri) uses a standard curve from 0.2 to 20 $\mathrm{mcU} / \mathrm{ml}$. To validate the assay, $100 \mathrm{U} / \mathrm{ml}$ of Glargine was diluted volumetrically with $6 \mathrm{~g} / \mathrm{dl}$ of Bovine Serum Albumin to final concentrations of 40, 20, 10, and 5 $\mathrm{mcU} / \mathrm{ml}$. These dilutions were prepared in triplicate and treated with 25\% PEG, and every dilution was then analyzed in duplicate using the Linco Research Free Insulin Extraction Procedure (PEG protocol). The percentage of cross-reactivity was calculated from a ratio of measured and nominal concentrations. The cross-reactivity was determined to be $94 \%$ at a standard concentration of 5 $\mathrm{mcU} / \mathrm{ml}$ (35 pmol/l); levels that correspond well with those observed in our study.

\subsection{Statistical Analysis}

The primary outcome variable of the study was the serum insulin concentration during the final 4 hours of the 24-hour study period. It was determined that ten subjects would be adequate to provide $80 \%$ power to detect a $49 \mathrm{pmol} / \mathrm{l}$ difference in serum insulin during the last 4 hours of the 24-hour study period between the once-daily 
and twice-daily insulin glargine regimens using a paired student's $t$-test with alpha equal to 0.05 . Secondary outcome variables include all insulin and glucose concentrations over the 24-hour period and the results of home CBG testing during the week prior to admission. Results from the once-daily and twice-daily insulin dosing regimens were compared using ANOVA and the paired Student's $t$ test, when appropriate. Area under the curve (AUC) for insulin and glucose during the last four hours of study was calculated using the trapezoidal rule. Results are reported as mean $\pm \mathrm{SD}$, except in the figures, which depict mean \pm SEM.

\section{RESULTS}

\subsection{Patient Characteristics}

Subjects had a mean age of $40 \pm 8$ years, a mean duration of diabetes of $16 \pm 10$ years, and a mean BMI of 31 $\pm 8 \mathrm{~m} / \mathrm{kg}^{2}$. All enrolled subjects were C-peptide negative (less than $1 \mathrm{pmol} / \mathrm{ml}$ ) during hyperglycemia 60 minutes after ingestion of 8 ounces of Boost ${ }^{\circledR}$ nutritional beverage (Nestle HealthCare Nutrition, Inc., Minnetonka).

\subsection{Serum Free Insulin Concentrations}

Figure 1 shows the mean free insulin concentrations obtained during the 24 hour study period. At baseline (0800), there was no statistical difference in serum free insulin levels between the once-daily and the twice-daily insulin glargine regimens (137 \pm 97 vs. $146 \pm 128 \mathrm{pmol} / \mathrm{l}$, respectively, $p=0.86$ ). Twelve hours after the first injection of insulin glargine, serum insulin levels in the once-daily treatment regimen were significantly higher than during the twice-daily treatment regimen (150 \pm 99 vs. $63 \pm 39 \mathrm{pmol} / \mathrm{l}, p=0.02)$. At the end of the 24-hour study period, there was no significant difference between the serum insulin levels in the once-daily and the twicedaily insulin glargine regimens $(70 \pm 56$ vs. $84 \pm 63$ $\mathrm{pmol} / \mathrm{l}, p=0.60$ ). Table 1 summarizes the AUC for insulin during the last four hours of the 24-hour study period.

\subsection{Plasma Glucose Concentrations}

At baseline (0800), there was no significant difference between the glucose levels in the once-daily or the twicedaily insulin glargine treatment regimens (86 \pm 35 vs. 97 $\pm 38 \mathrm{mg} / \mathrm{dl}$ and $4.8 \pm 1.9$ vs. $5.4 \pm 2.1 \mathrm{mmol} / \mathrm{l}$, respectively, $p=0.54)$. Twelve hours after the first insulin glargine injection, glucose concentrations were not significantly different despite a statistical difference in serum insulin levels ( $83 \pm 53$ vs. $108 \pm 58 \mathrm{mg} / \mathrm{dl}$ and $4.6 \pm$ 2.9 vs. $6.0 \pm 3.2 \mathrm{mmol} / \mathrm{l}, p=0.30$ ). At the end of the 24-hour study, there was no statistical difference between the plasma glucose levels in the once-daily and the twice-daily insulin glargine regimens (119 \pm 44 vs. $134 \pm$

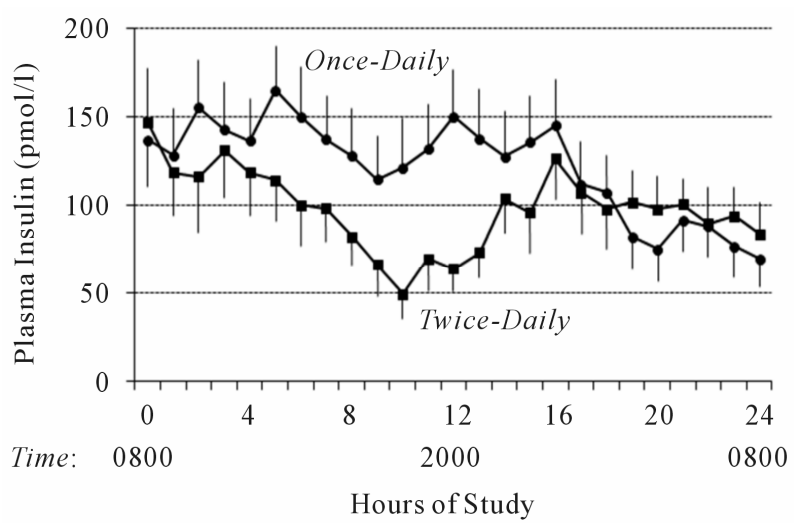

Figure 1. Serum free insulin concentrations following oncedaily (solid circles) and twice-daily (solid squares) insulin glargine injection during a 24-hour fast. To convert to $\mathrm{mcU} / \mathrm{ml}$, divide by 7 .

Table 1. Comparison of serum free insulin concentration AUC during the final four hours of a 24-hour fast between once-daily and twice-daily insulin glargine injection.

\begin{tabular}{cccc}
\hline $\begin{array}{c}\text { Time } \\
\text { Period }\end{array}$ & $\begin{array}{c}\text { Once Daily } \\
\text { Insulin Glargine } \\
\text { (pmol/l-time) }\end{array}$ & $\begin{array}{c}\text { Twice Daily } \\
\text { Insulin Glargine } \\
(\text { pmol/lttime })\end{array}$ & $p$-value \\
\hline $0400-0800$ & $53 \pm 37$ & $48 \pm 37$ & 0.38 \\
$0600-0800$ & $25 \pm 17$ & $22 \pm 15$ & 0.24 \\
$0700-0800$ & $13 \pm 9$ & $11 \pm 7$ & 0.24 \\
\hline
\end{tabular}

$48 \mathrm{mg} / \mathrm{dl}$ and $6.6 \pm 2.4$ vs. $7.4 \pm 2.7 \mathrm{mmol} / \mathrm{l}, p=0.24$ ). Figure 2 depicts the mean glucose concentrations during the 24 hour study period, and Table 2 summarizes the AUC for glucose during the last four hours of the 24hour study period.

Nine of ten subjects recorded and returned home capillary blood glucose results for the week prior to admission. As shown in Figure 3, there was no significant difference between mean home CBG values recorded during the morning before breakfast $(174 \pm 74$ vs. $168 \pm 72$ $\mathrm{mg} / \mathrm{dl}$ and $9.7 \pm 4.1$ vs. $9.3 \pm 4 \mathrm{mmol} / \mathrm{l}, p=0.64)$ or in the evening before supper with once-daily or twice-daily insulin glargine dosing (169 \pm 92 vs. $191 \pm 104 \mathrm{mg} / \mathrm{dl}$ and $9.4 \pm 5.1$ vs. $10.6 \pm 5.8 \mathrm{mmol} / \mathrm{l}$, respectively, $p=$ 0.27).

\section{CONCLUSIONS}

Pharmacodynamic studies have suggested that there may be a window of relative insulinopenia with oncedaily administration of insulin glargine because the duration of action for insulin glargine insulin being approximately 22 hours [2,3]. Furthermore, patients often report a rise in glucose concentration in the hours just prior to their next scheduled insulin glargine injection. The present study addressed the question of whether or not 
Table 2. Comparison of plasma glucose concentration AUC during the final four hours of a 24-hour fast between once-daily and twice-daily insulin glargine injections.

\begin{tabular}{cccc}
\hline $\begin{array}{c}\text { Time } \\
\text { Period }\end{array}$ & $\begin{array}{c}\text { Once Daily } \\
\text { Insulin Glargine } \\
(\mathrm{mg} / \mathrm{dl} \text {-time })\end{array}$ & $\begin{array}{c}\text { Twice-Daily } \\
\text { Insulin Glargine } \\
(\mathrm{mg} / \mathrm{dl} \text {-time })\end{array}$ & $p$-value \\
\hline $0400-0800$ & $218 \pm 78$ & $211 \pm 86$ & 0.74 \\
$0600-0800$ & $118 \pm 43$ & $105 \pm 43$ & 0.17 \\
$0700-0800$ & $62 \pm 23$ & $55 \pm 22$ & 0.11 \\
\hline
\end{tabular}

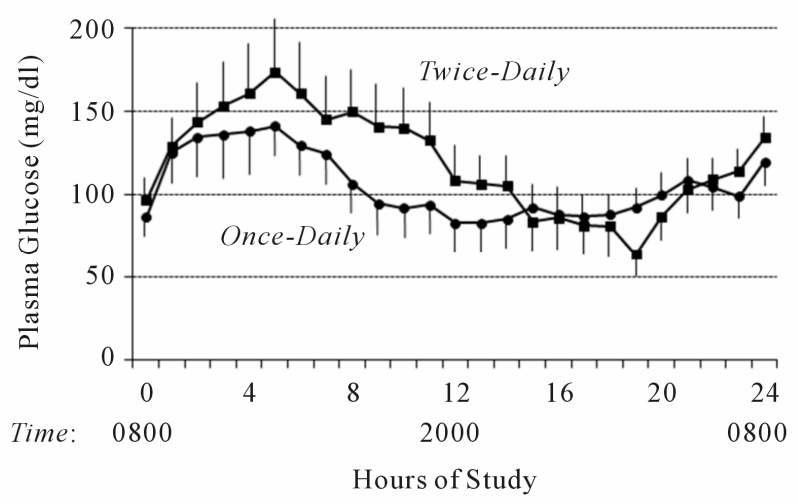

Figure 2. Mean plasma glucose concentrations following oncedaily (solid circles) and twice-daily (solid squares) insulin glargine injection during a 24-hour fast. To convert to $\mathrm{mmol} / \mathrm{l}$, multiply by 0.05551 .

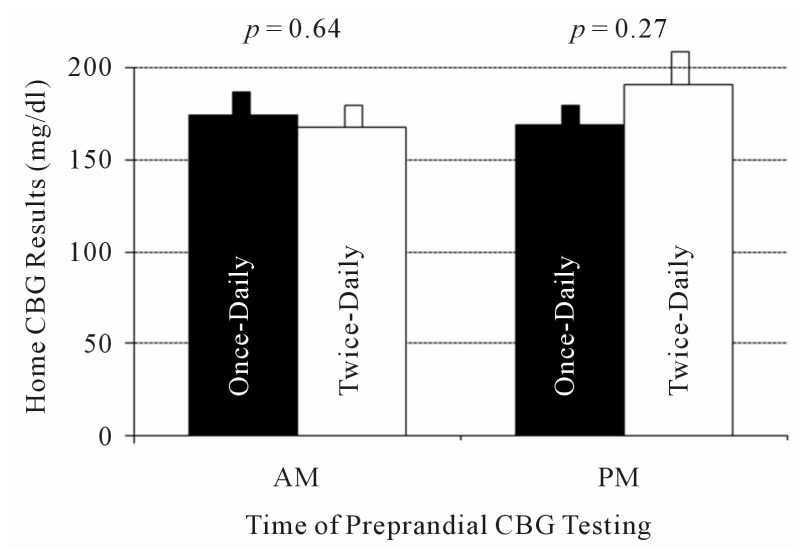

Figure 3. Morning and evening home capillary blood glucose (CBG) concentrations do not differ between once-daily (solid bars) and twice-daily (empty bars) insulin glargine dosing. To convert to $\mathrm{mmol} / \mathrm{l}$, multiply by 0.05551 .

administering insulin glargine as a once-daily full-dose or as twice-daily half-doses 12 hours apart affects serum insulin concentrations during the final hours of a 24-hour study period. We used a fasting model to eliminate the confounding factor of rapid acting insulin. We also held long-acting insulin on the day prior to study in attempt to eliminate carry-over effects from the previous days' insulin injections. In addition, we used a highly sensitive insulin assay in attempt to optimize determination of insulin glargine concentrations.

These data did not show improved insulinization at the end of a 24 hour period with twice-daily insulin glargine compared to once-daily insulin glargine dosing. Moreover, these data contradict the case report by Clement $e t$ al., which demonstrated improved glycemic control with twice-daily insulin glargine injections in an uncontrolled report [4]. It should be noted that the current study did find a statistical difference between the treatment regimens in insulin concentrations at 12 hours, but at that point only half of the total daily insulin glargine dose had been given in the twice-daily regimen. This finding is likely to be accentuated by the fact that insulin glargine was withheld on the day prior to admission because, whereas in a more clinically realistic setting, there would have been overlap in insulin concentrations from the previous day's insulin glargine dose.

Albright et al. demonstrated increased glycemic control with twice-daily insulin glargine administration in type 1 diabetics, but a nearly 70\% increase in the insulin glargine dose was required to achieve that result [7]. Further, twice-daily split doses were given only if titration of bedtime insulin glargine resulted in morning hypoglycemia or if subjects had persistent elevation of afternoon blood glucose values that could not be corrected with bolus titration. Thus, twice-daily dosing allowed continued titration of the insulin glargine dose. In the present study, subjects received equivalent total daily doses of insulin glargine during both the once-daily and twice-daily treatment regimens, and no benefit to the twice-daily approach was demonstrated.

Ashwell et al. demonstrated that serum free insulin levels were increased at the end of a 24-hour study period in fifteen type 1 diabetics using a once-daily insulin glargine regimen compared to the same patients using a twice-daily regimen [8]. However, the subjects were not fasting and continued to receive rapid-acting insulin with meals, thereby confounding interpretation of that study. The protocol described in this report has the advantage of isolating the effect of the basal insulin by fasting the subjects to allow for the omission of rapid acting insulin injections.

This study is limited by the relatively small number of study participants. Additionally, given that subjects remained in a fasting state during the inpatient admissions, we cannot definitively comment on the utility of twicedaily insulin glargine when used in combination with rapid acting pre-prandial insulin. Furthermore, we cannot definitively comment about the longer term efficacy of twice-daily insulin glargine versus once-daily insulin glargine or the effect of such a regimen on A1c values. Based upon our prospective power analysis, this study excludes a difference of $49 \mathrm{pmol} / \mathrm{l}$ or greater between 
once- and twice-daily insulin glargine. Moreover, posthoc statistical analysis eliminates the possibility of a difference in insulin concentrations $>20 \mathrm{pmol} / \mathrm{l}$ between the two modes of insulin dosing with $95 \%$ confidence.

In conclusion, insulin and glucose concentrations were not significantly different at the end of a 24-hour period when equivalent total doses of insulin glargine were given as a once-daily injection as compared to twicedaily injections consisting of two half-doses 12 hours apart during a 38 hour fast. The common practice of using twice-daily injections as a strategy to address the waning effectiveness of insulin glargine at the end of its activity period is not supported by these data.

\section{ACKNOWLEDGEMENTS}

This study was supported by the University of New Mexico General Clinical Research Center (GCRC; M01 \# RR000997-35) and the University of New Mexico Clinical and Translational Research Center (5KL2RR031976-02, 5UL1RR031977-02).

The insulin glargine used for this study was supplied as a gift from Sanofi Aventis, Inc.

None of the authors have any duality of interest to declare regarding this study.

\section{REFERENCES}

[1] Hirsch, I. (2005) Insulin analogues. The New England Journal of Medicine, 352, 174-183. doi:10.1056/NEJMra040832
[2] Lepore, M., Pampanelli, S., Fanelli, C., et al. (2000) Pharmacokinetics and pharmacodynamics of subcutaneous injection of long-acting human insulin analog glargine, NPH insulin, and ultralente human insulin and continuous subcutaneous infusion of insulin lispro. Diabetes, 49, 2142-2148. doi:10.2337/diabetes.49.12.2142

[3] Heinemann, L., Linkeschova, R., Rave, K., et al. (2000) Time-action profile of the long-acting insulin analog insulin glargine (HOE901) in comparison with those of NPH insulin and placebo. Diabetes Care, 23, 644-649. doi:10.2337/diacare.23.5.644

[4] Clement, S. and Bowen-Wright, H. (2002) Twenty-four hour action of insulin glargine (Lantus) may be too short for once-daily dosing: A case report. Diabetes Care, 25, 1479-1480. doi:10.2337/diacare.25.8.1479-a

[5] American Diabetes Association. (2008) Standards of medical care in diabetes-2008. Diabetes Care, 31, S12S54.

[6] Owen, W.E. and Roberts, W.L. (2004) Cross-reactivity of three recombinant insulin analogues with five commercial insulin immunoassays. Clinical Chemistry, 50, 257-259. doi:10.1373/clinchem.2003.026625

[7] Albright, E.S., Desmond, R. and Bell, D.S.H. (2004) Efficacy of Conversion from bedtime NPH insulin injecttion to once- or twice-daily injections of insulin glargine in type 1 diabetic patients using basal/bolus therapy. Diabetes Care, 27, 632-633. doi:10.2337/diacare.27.2.632

[8] Ashwell, S.G., Gebbie, J. and Home, P.D. (2006) Twicedaily compared to once-daily insulin glargine in people with type 1 diabetes using meal-time insulin aspart. Diabetic Medicine, 23, 879-886. doi:10.1111/j.1464-5491.2006.01913.x 